\title{
Orbital Structure Analysis in Molecular Electronics
}

\author{
Manas Ranjan Pradhan \\ IBS, Hyderabad \\ Survey No.156/157, Dontanaplli, ShankarPalli
}

\author{
E.G.Rajan \\ MGNIRSA, Hyderabad \\ Gagan Mahal road, Domalguda
}

\begin{abstract}
The objective of this research work is to find out the orbital structure effect of the molecules used in a molecular wire of polyphenylene chain which facilitate the electron flow in a molecular surface and in turn gives electronic properties. Taking molecular rectifier diode as the part of study, we have analyzed the hybridization property maintained among molecules where electrons get flowed from donor part to acceptor part with insulator as intermediate. The whole of the study takes place as donor part molecular orbital, acceptor part molecular orbital and the insulator part molecular orbital and how does it affect to the whole single molecule as Donor-Insulator-Acceptor. The use of Density function theory and Non equilibrium green's function are mentioned to study the voltage $\sim$ current characteristics and detailed quantitative model will be put in our next research work.
\end{abstract}

Key words: Molecular Electronics, Molecular Orbital, Atomic Orbital

\section{INTRODUCTION}

The general principle of analyzing the electron liberation in a surface is analyzed by photoelectric effect. This liberation of electrons from matter (here it is molecular wire as a whole) happens under the influence of sufficiently high frequency electromagnetic raditation, particularly ultraviolet and X-rays. Assuming the molecular surface is photosensitive; the theory of electron conductivity signifies the existence of free electrons within the metals. From the viewpoint of photoelectric itself, the liberation of electrons by the electric field of light wave is easily understood [Fig.1]. The building blocks of molecular electronics are single or small pakets of molecules [1]. A number of molecular electronics based digital circuits have already been reported in different research work worldwide. If we restrict our studies only to molecular diodes there are two types of molecular diodes: rectifying diodes and resonant tunneling diodes which can both be used in realizing monomolecular digital logic circuits [2]. The whole molecules involved in designing the molecular diode has taken as monomolecular and the properties can only study with a combination of different molecular orbital structure. The study confined here is designing molecular surface with polyphenylene-based chains. The polyphenylene chains are nothing but the chain of interlinking benzene molecules. This chain of benzene along with subsidiary donor group, acceptor group and insulator group analyzed the electronics and electrical properties of molecular surface. Here we have considered a single molecular surface consisting of donor, acceptor and insulator. The electron flows happens from donor side to acceptor side with insulator intermediate. The electrical or electronic properties get analyzed only if we analyze the orbital features of all the molecular orbital involves. The molecular orbital features and its wave properties generally get studied by quantum mechanics and helps in analyzing the energy at different states starting from ground state of molecular combination to most excited state. With different voltage applied with internal resistor as insulator group molecule and external register, we will able to analyze variation of current with intensity of light (X-rays) and variation of current with negative potential at collector side. We can also analyze the stopping potential for kinetic energy study of most energetic electron and how does it affect molecular energy. 


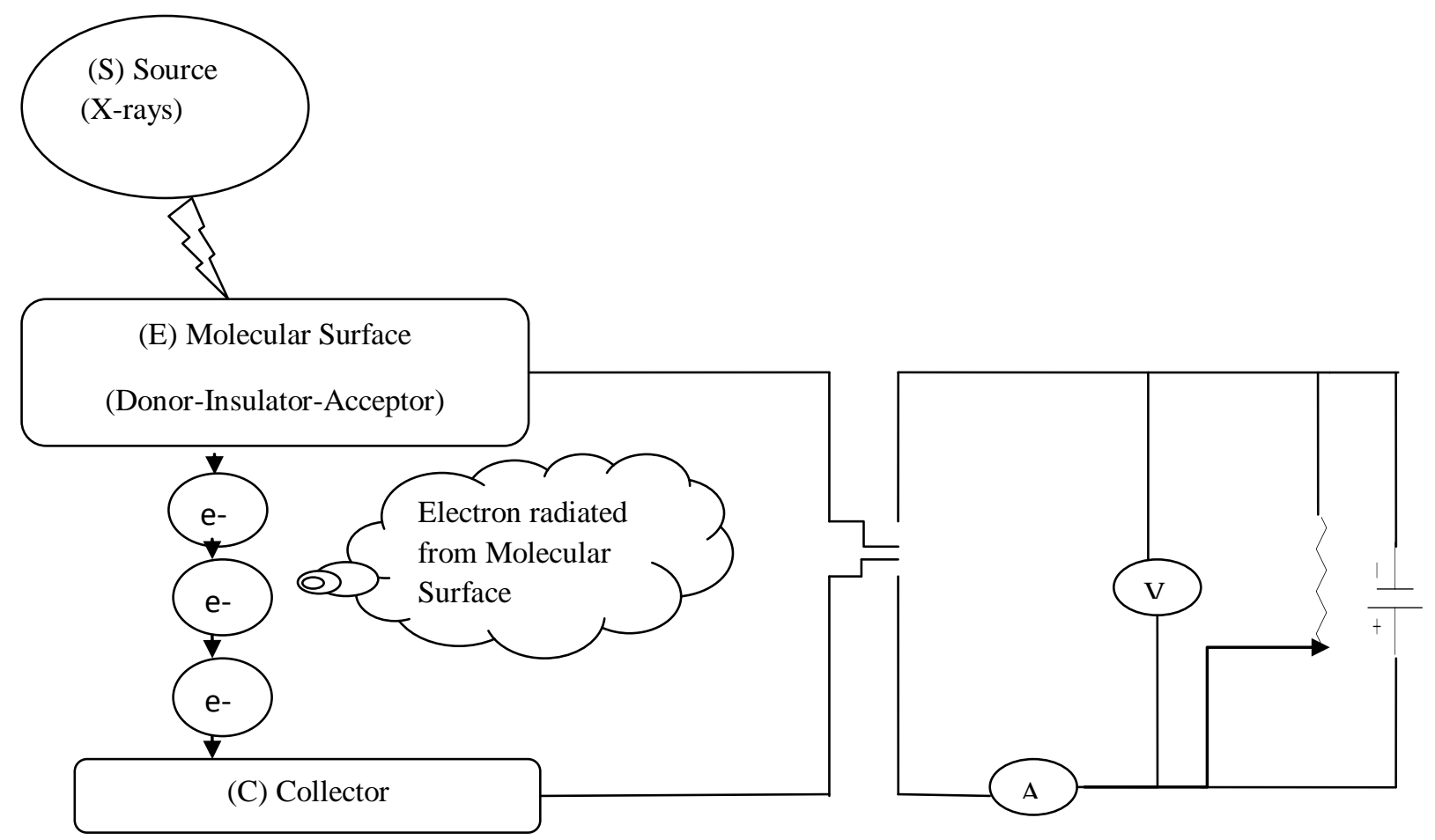

Fig1.Investigating a Molecular Surface

\section{MOLECULAR DIODE DESIGN}

The structure of the mono-molecular rectifying diode proposed in [3] is shown in Fig. 2 and its schematic representation is shown in Fig. 3.

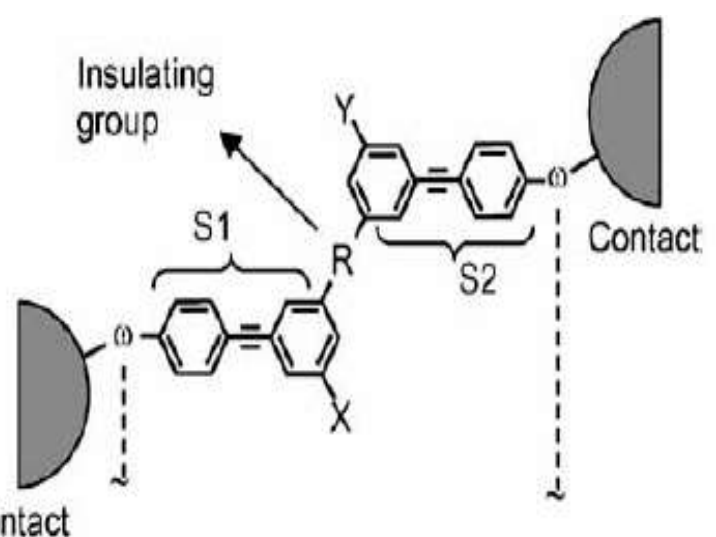

Fig.2 Structure of Rectifying Molecular Diode (mono-molecular diode). [Refer 1]

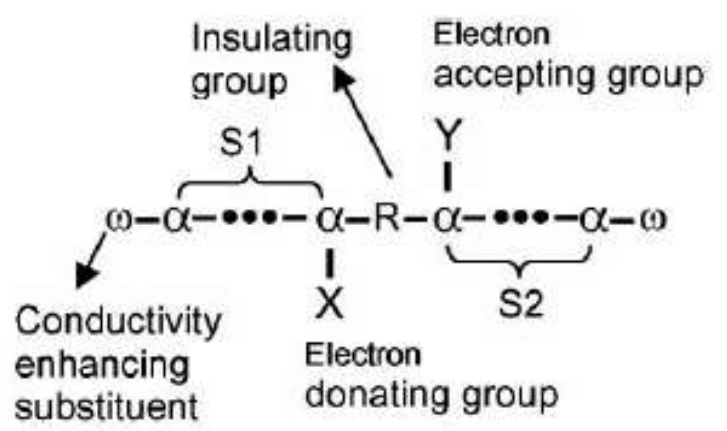

Fig.3 Schematic Representation of Rectifying Molecular Diode. [Refer 1]

The circuit representation of a diode logic AND gate is shown in (Fig. 4) and the schematic representation of the Diode logic molecular AND gate is shown in (Fig. 5). 


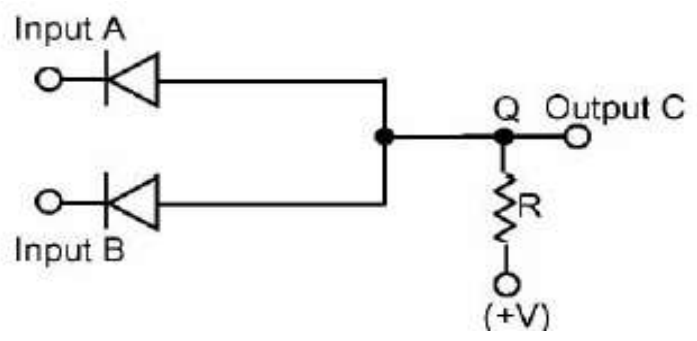

Fig.4 Circuit Diagram of AND gate

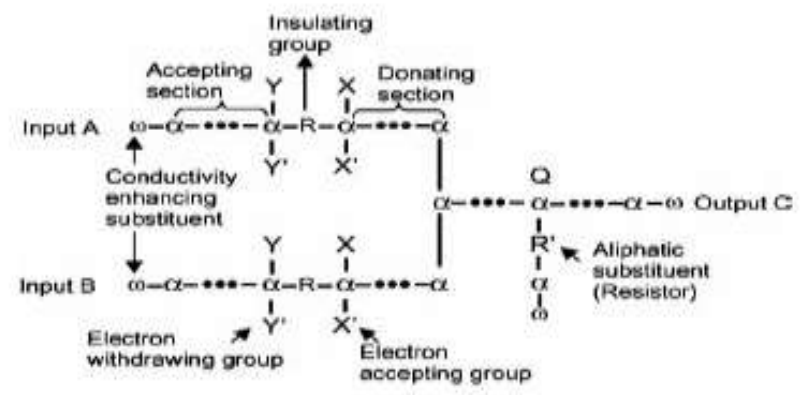

Fig.5 Schematic Representation of AND gate

A molecular diode contains two terminals and functions like a semiconductor $\mathrm{p}-\mathrm{n}$ junction and has electronic states as conductive state $(\mathrm{ON})$ and less conductive state $(\mathrm{OFF})$. As per Aviram and Ratner suggestion, electron-donating constituents make conjugated molecular groups having a large electron density $(\mathrm{N}$ type) and electron withdrawing constituents make conjugated molecular groups poor in electron density (P-type). A non-centro symmetric molecule having appropriate donor and acceptor linked with an $\sigma$-bridge (may act as an insulator or internal register) and connect with suitable electrodes will conduct current only in one direction - acting as a rectifier. In this analysis of Aviram and Ratner in a $D$ (donor)- $\sigma$ (insulator)- $A$ (acceptor) molecule, the lowest unoccupied molecular orbital (LUMO) and highest occupied molecular orbital (HOMO) can be aligned in such a way that electronic conduction is possible only in one direction making it function like a molecular diode [1].

\section{STRUCTURE ANALYSIS OF MOLECULE}

Referring Fig.2, we have used S1 as donate group and S2 as acceptor group in a single molecule of polyphenylene chain. S1 and S2 are typically benzene constitute molecule. In a polyphenylene chain all these benzene molecules form chain and the electrical conductivity properties can be tested with different donor group $\mathrm{X}$ and acceptor group $\mathrm{Y}$. Referring to Fig.3 the contact metal (w) may taken any inorganic metal like gold, uranium, aluminium etc.The electrical properties and flow of electrons generally depict out if the valence electrons available for the molecule.
The donating group element can be $(\mathrm{X})=\left(-\mathbf{N H}_{2},-\mathbf{O H},-\mathbf{C H}_{3}\right.$, $\mathrm{CH}_{2} \mathrm{CH}_{3}$, etc.)

The different types of Acceptor group element that we take as (Y) $=\left(-\mathrm{NO}_{2},-\mathrm{CH},-\mathrm{CHO},-\mathrm{NC}\right.$ etc. $)$

The different types of Insulator group element that we take as (R) $=\left(-\mathrm{CH}_{2-}^{-},-\mathrm{CH}_{2}-\mathrm{CH}_{2^{-}}\right.$, etc. $)$

The combination of $\mathrm{X}, \mathrm{Y}$ and $\mathrm{R}$ in molecular conduction creates an uncertainty of electron flow.

The uncertainty arrives as

a) Fixing one $\mathrm{X}$ (say $-\mathrm{NH} 2)$, we may have varieties of $\mathrm{Y}\left(\left(-\mathrm{NO}_{2}\right.\right.$, $\mathrm{CH},-\mathrm{CHO},-\mathrm{NC})$ and $(\mathrm{R})=\left(-\mathrm{CH}_{2^{-}},-\mathrm{CH}_{2}-\mathrm{CH}_{2^{-}}\right)$

b) Fixing one $\mathrm{R}$ (say $-\mathrm{CH} 2)$, we may have varieties of $\mathrm{Y}\left(-\mathrm{NO}_{2}\right.$, $\mathrm{CH},-\mathrm{CHO},-\mathrm{NC})$ and $(\mathrm{X})=\left(-\mathrm{NH}_{2},-\mathrm{OH},-\mathrm{CH}_{3},-\mathrm{CH}_{2} \mathrm{CH}_{3}\right)$

c) Fixing one $\mathrm{Y}$ (say $-\mathrm{NO} 2)$, we may have varieties of $(\mathrm{X})=(-$ $\left.\mathrm{NH}_{2},-\mathrm{OH},-\mathrm{CH}_{3},-\mathrm{CH}_{2} \mathrm{CH}_{3}\right)$ and $(\mathrm{R})=\left(-\mathrm{CH}_{2}-,-\mathrm{CH}_{2}-\mathrm{CH}_{2}-\right)$

Taking an Example where S1 and S2 are two benzene chain and $\mathrm{X}$ taken as $\mathbf{N H}_{2}$ and $\mathrm{Y}$ taken as $\mathbf{N O}_{2}$ with insulator as $\mathbf{C H}_{2}-\mathrm{CH}_{2}$. It forms now one molecular orbital of Benzene-Benzene of $\mathrm{S} 1$ for Donor group, different molecular orbital for benzene-benzene of S2 and another molecular orbital for insulator.

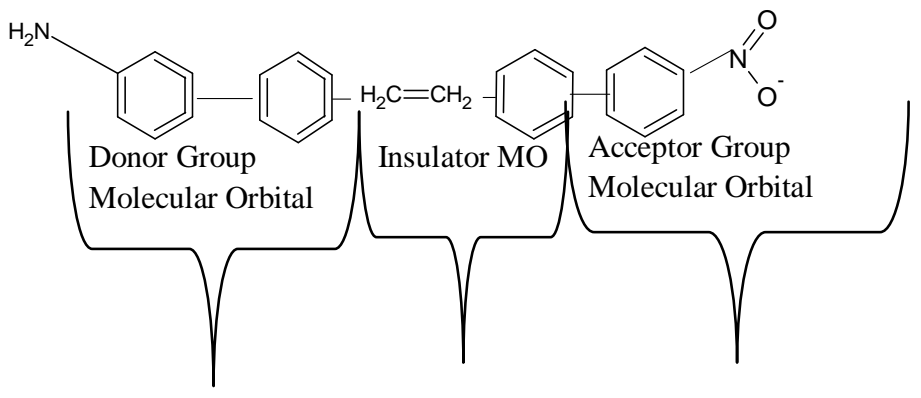

Fig6.Classifying Different Molecular Orbital in a Molecular Rectifying Diode

The molecule as a whole maintaining the valence of each atom will be $\left(\mathrm{H}_{2} \mathrm{~N}-\mathrm{C}_{6} \mathrm{H}_{3}-\mathrm{C}_{6} \mathrm{H}_{3}-\mathrm{CH}_{2}-\mathrm{CH}_{2}-\mathrm{C}_{6} \mathrm{H}_{3}-\mathrm{C}_{6} \mathrm{H}_{3}-\mathrm{NO}_{2}\right)$<smiles>NC1=CCC2=C(C1)c1ccccc12</smiles>

biphenylen-2-amine

Fig7.Donor Molecule (H2N-C6H3-C6H4) 
<smiles>O=C=Nc1cccc2c1C1=C3C=CC=CC3C12[18OH]</smiles>

Fig8.Acceptor Molecule (C6H4-C6H3-NO2)<smiles>O=[N+]([O-])c1cccc2c1-c1ccccc1-2</smiles>

Fig9.Same as Fig8 with different structure to NO2

$$
\begin{gathered}
\mathrm{H}_{2} \mathrm{C}=\mathrm{CH}_{2} \\
\text { ethene }
\end{gathered}
$$

Fig10.Insulator

As shown in fig.7, the donor group as a molecule is called as biphenylen-2-amine. As per fig.8; the whole molecule for acceptor is 1-nitrosobiphenylene or 1-nitrobiphenylene. Similarly the insulator as ethene. The analysis can be done whether biphenylen-2-amine, 1-nitrosobiphenylene or 1-nitrobiphenylene and ethene can show donor properties, acceptor properties and insulating properties respectively in a single molecule of chain $\left(\mathrm{H}_{2} \mathrm{~N}-\mathrm{C}_{6} \mathrm{H}_{3}-\mathrm{C}_{6} \mathrm{H}_{3}-\mathrm{CH}_{2}-\mathrm{CH}_{2}-\mathrm{C}_{6} \mathrm{H}_{3}-\mathrm{C}_{6} \mathrm{H}_{3}-\mathrm{NO}_{2}\right)$.

Now the conducting properties of donor \& acceptor and the insulating properties of bridge molecule can be studied provided we study the molecular orbital features and hybridization properties. These properties can be studied in two ways; one is by changing the molecules for $\mathrm{X}, \mathrm{Y}, \mathrm{R}$ or changing the position of $\mathrm{X}$ and $\mathrm{Y}$ at different carbon atoms of benzene. This also facilitates different bond types.

I) By Changing the molecules: by changing the different $\mathrm{X}, \mathrm{Y}, \mathrm{R}$ the different molecules will give different Molecular Orbital result and determine the electron density in dodnor, acceptor and insulator region. If we look at the above molecular wire and the donor molecule, the name will change if we take any other molecule different from $\mathrm{NH}_{2}$ like $-\mathbf{O H},-\mathbf{C H}_{3},-\mathbf{C H}_{2} \mathbf{C H}_{3}$ etc. These changes make the molecular orbital properties also changed. Similarly the acceptor molecular properties will change if we have different other molecule different from $\mathrm{NO}_{2}$ like - $\mathrm{CH}$, $\mathrm{CHO}$, - NC etc.

II) By changing the position of $\mathrm{X}$ and $\mathrm{Y}$ around different carbon atom position of benzene chains, gives a randomness for the atom as well as for molecule to fix at any point matching to valence condition and molecular orbital hybridization properties. Taking same set of $\mathrm{X}, \mathrm{Y}$ and $\mathrm{R}$ but changing the position of $\mathrm{X}$ and $\mathrm{Y}$ at different carbon atom, it will create different structure to the molecule as well as different naming which in other sense affects the molecular orbital properties.

\subsection{Molecule and Atomic Properties}

Discussing about the donor molecule the $\mathrm{X}$ which is $\mathrm{NH}_{2}$, is itself a molecule derived from atoms $\mathrm{N}$ and $\mathrm{H}$ and the molecular orbital formed for $\mathrm{NH}_{2}$ depends on atomic properties like atomic number, electronic configuration, electronic affinity, ionization potential and electron density. $\mathrm{NH}_{2}$ formed assuming the bond properties between $\mathrm{N}$ and H.Now $\mathrm{NH}_{2}$ is revolving around any carbon atom of benzene seems like carbon atom act as the central controlling atom and $\mathrm{NH}_{2}$ is revolving around it. If we look at benzene molecule, it is a combination of atomic orbital of Carbon and Hydrogen. One benzene joins with another in a polyphenylene chain makes the structure as a conjugate molecule. The concern is to study the electronics properties in many electrons, many atoms and many molecules acting as a single molecule. The factors that influence the atomic properties are the shape of molecules, bond angle, lone pairs of electrons, bonding \& antibonding, atomic orbital shapes, directionality of bonds, hybridization, resonance and intermolecular forces. The quantum mechanical property of molecule as a whole makes us to know the way to find the energy states of the molecule or subsidiaries that used for molecular electronics.

According to modern theory, an atom consists of a central nucleus of positive charges surrounded by negatively charged particles called electrons. The charge of electron is equal and opposite to that of a proton. Under ordinary condition the number of electrons is equal to the number of protons. The electrons in an atom revolve around the nucleus in different orbits or paths. The number and arrangement of electrons in any orbit is determined by the rule, which tells the number of electrons in any orbit is given by $2 \mathrm{n}^{2}$, where $\mathrm{n}$ is the number of the orbit. The principle it follows are the last orbit can not have more than 8 electrons and the last but one can not have more than 18 electrons. Some important properties of electron are: Charge of electron $\left(\mathrm{e}=1.602 * 10^{-19}\right.$ coulomb), Mass of an electron $\left(\mathrm{m}=9.0 * 10^{-31} \mathrm{~kg}\right)$, Radius of an electron, $\mathrm{r}=1.9 * 10^{-15}$ metre and the ratio e/m of an electron is $\left(1.77^{*} 10^{-11}\right)$ coulombs $/ \mathrm{kg}$. This means that mass of an electron is very small as compared to its charge. It is due to this 
property of an electron that it is very mobile and is greatly influenced by electric or magnetic field. An electron moving around the nucleus possesses two types of energies viz.kinetic energy due its motion and potential energy due to charge on the nucleus. The total energy is sum of these two. The energy of an electron increases as its distance from the nucleus increases. The last orbit electrons play an important role in determining the physical, chemical and electrical properties of a material. When the number of valence electrons of an atom is less than 4, it is a metal and a conductor. When the number of valence electrons of an atom is more than 4, the material is a non-metal and an insulator. When the number of an valence electrons of an atom is 4 , the material has both metal and non-metal properties and called as semi-conductor. The valence of an atom means the number of electrons it needed to fulfill the saturation of orbit. It is needed for determining the bond. H-hydrogen is a special case of gas as it has single electron in $1^{\text {st }}$ orbit itself. C-carbon has exactly 4 electronsso semi-conductors. Oxygen has 6 electrons so act as a non-metal and insulator. N-has 5 electrons so act as an insulator.

The valence electrons of different materials possess different energies. The greater the energy of a valence electron, the lesser it is bound to the nucleus. The loosely attached valence electrons move at random within the material and are called free electrons. A conductor is a substance with large number of free electrons. When potential difference is applied across a conductor, the free electrons move towards the positive terminal of supply, constituting electric current [fig.1]. An insulator is a substance, which has practically no free electrons at ordinary temperature. Therefore an insulator does not conduct current under the influence of potential difference. The electrons are free only to the extent that they may transfer from atom to another within the surface to provide electron emission. But these properties get on change if we think about molecular orbit.

\subsection{Hybridization}

In the processing of forming molecules from atoms, a linear combination of atomic orbitals of atoms is carried out to form a new set of atomic orbitals based on the requirements of bond formation and the influence of neighboring atoms. The linear combination of atomic orbitals of an atom is called as hybridization of atomic orbitals. Hybridization does not change the number of orbitals, but their orientation and distribution are changed. Hybrid orbitals have similar properties as atomic orbitals.

We can say $\Psi=\mathrm{C}_{1} \Phi_{1}+\mathrm{C}_{2} \Phi_{2}+\ldots$, where $\Psi=$ Molecular Orbital or hybrid orbital $\Phi 1, \Phi_{2}$ are atomic orbitals. It will achieve the properties of orthogonal and normalization.

If the probability for an orbital is given by $\Psi^{2}$ and the integral $\int \Psi^{83} \Psi \mathrm{d} \tau=1$, then for Molecular Orbital's

$$
\begin{aligned}
& \Psi^{2}=\mathrm{C}_{1}{ }^{2} \Phi_{1}{ }^{2}+\mathrm{C}_{2}^{2} \Phi_{2}^{2}+2 \mathrm{C}_{1} \mathrm{C}_{2} \Phi_{1} \Phi_{2} \\
& \text { Or } \Psi^{2}=\mathrm{C}_{1}^{2} \mathrm{P}_{1}+\mathrm{C}_{2}^{2} \mathrm{P}_{2}+2 \mathrm{C}_{1} \mathrm{C}_{2} \mathrm{P}_{12}
\end{aligned}
$$

Where $\mathrm{P}$ represents the atomic orbital probability.

Integrating it gives $\int \Psi^{2} d \tau=\mathrm{C}_{1}+\mathrm{C}_{2}+2 \mathrm{C}_{1} \mathrm{C}_{2} \mathrm{~S}_{12}$ where $S_{12}$ is the overlap integral, the sign of which is dependent on the coordinate system. $C_{1}^{2}$ and $C_{2}^{2}$ give the $\Phi_{1}$ and $\Phi_{2}$ character. The types of bonds arise from whether P12 is based on the interaction of one lobe from each atomic orbital, two lobes or four lobes. The sigma bond is formed with 1 lobe and the atomic orbitals used are (s, s; s, p; p, p; d, d). Similarly pi bond is formed with 2 lobes and the atomic orbitals (p, p; d, d; p, d). The delta bond formed with 4 lobes and atomic orbitals are (d, d).

The value of $2 \mathrm{C}_{1} \mathrm{C}_{2} \mathrm{P}_{12}$ in the region of the bond axis indicates the type of bonding interaction. If $2 \mathrm{C}_{1} \mathrm{C}_{2} \mathrm{P}_{12}>0$, it is bonding, for $2 \mathrm{C}_{1} \mathrm{C}_{2} \mathrm{P}_{12}<0$, it is anti-bonding and in case it is $=0$, it is nonbonding. Succesively higher energy orbitals have Molecular node symmetrically placed, and increasing in number by one, in order from most stable to least stable.

The most common application of Molecular Orbital theory in polyatomic molecules is the combination non-hybrid atomic orbitals. The electrons in these molecular orbitals are delocalized over the whole molecule and not between two atoms. The concept of delocalization is effective in discussing the excited state of molecules, their ionization potentials and spectroscopic properties. According to molecular orbital theory, electrons are placed in molecular orbitals. Discrete bonds are not formed between atoms; electrons may "roam" over multiple nuclei. The hybridization models exist with $\mathrm{sp}^{3}, \mathrm{sp}^{2}$ and $\mathrm{sp}$ orbitals. Considering models of bonding $\mathrm{sp}^{3}$ hybridization occurs for atoms having four groups in a tetrahedral arrangement. Similarly $\mathrm{sp}^{2}$ hybridization occurs for atoms having three groups in a trigonal (planar) arrangement and $\mathrm{sp}$ hybridization occurs having two groups in a linear arrangement. Others are like trigonal bipyramidal as sp3d and octahedral as sp3d2.Group may be an electron pair also. Considering bonds we have ionic, covalent and polar covalent bond. The various types of bonding affect the shape, physical properties and reactivity. Hybridization and molecular geometry are closely correlated. The covalent bond has a definite direction in space (compare to ionic bonds). The directionality of covalent bonding is responsible for molecular shape. Orbitals can overlap in two ways-energy can add called bonding orbital or energy can subtract called anti-bonding orbital.Two types of molecular orbitals exist; one is sigma- $\partial$ orbitals (can be bonding or antibonding) comes from s orbitals and $\mathrm{p}$ orbitals that overlap end-toend. The other one is $\prod$ orbitals (can be bonding or anti-bonding) comes from $\mathrm{p}$ orbitals that overlap side-to-side. Electrons will fill by Puali exclusion principle and hund's rule. 


\subsubsection{Benzene Rings and Polyphenylene Chain}

$\mathrm{X}$-ray studies show that a Benzene molecule is a flat (planar) molecule. All carbon and hydrogen atoms lie in the same plane. It has a regular hexagon structure with all six-carbon atoms lying at the corners; each carbon atom is bonded to three other atoms. All carbon-carbon bond lengths are equal at $139 \mathrm{pm}$. All CC angles (or $\mathrm{CH}$ angles) are equal at 120 degree. These results indicate that each carbon atom in benzene molecule is sp2 hybridized. All sp2 hybrid orbitals lie in the same plane (the plane of the carbon atoms) and are oriented towards the corners of an equilateral triangle. Thus, each carbon in benzene has three sp2 hybrid orbitals lying in the same plane and one -unhybridized ' $\mathrm{p}$ ' orbital. Polyphenylene wires conduct because of the conjugated pi-orbital lying above and below the plane of the molecule forming a channel for electron transport when the wire is based by an external voltage. So Benzene is a flat ring of $\mathrm{sp} 2$ hybrid carbon atoms with their unhybridzed $\mathrm{p}$ orbitals all aligned and overlapping. The conjugation and delocalization of the electrons in benzene gives this compound greater stability than nonconjugate cycles. In benzene, the six $\mathrm{C}-\mathrm{C}$ bonds are equivalent and there is no distinct single and double bonds. It is difficult to explain the conjugate properties by altering localized single and double bonds. The simple and effective way is the Huckel Molecular Orbital Method.

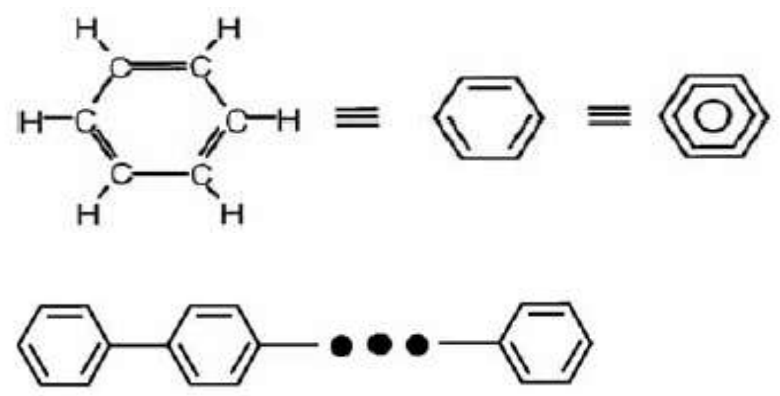

Fig.11 Benzene structure and polyphenylene chain

\subsubsection{Donor Molecule (H2N-C6H3-C6H4) Properties} study-

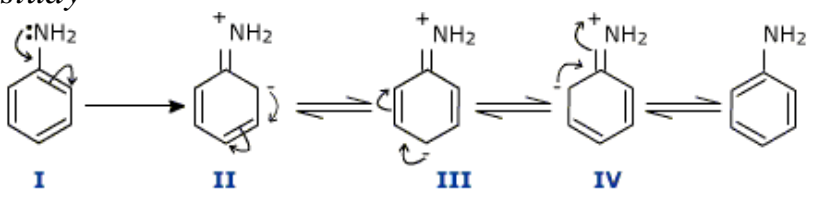

Fig. 12 -biphenylen-2-amine ((Drawn using ACID/ChemSketch Software)

Amiline is a hybrid of the above resonating structures. Thus the lone pair of electrons on nitrogen is withdrawn from it and is being partially shared with the benzene ring. Thus, in aniline the electron donating capacity of nitrogen for protonation is considerably decreased as compared to that of ammonia and aliphatic amines. Hence aniline is a weaker base than aliphatic amines and ammonia. In amines, there is a lone pair of electrons on nitrogen. The tendency of nitrogen to share these electrons with acids is responsible for the basic character of amines.

\subsubsection{Insulator $\left(\mathrm{H}_{2} \mathrm{C}-\mathrm{CH}_{2}\right)$ properties study}

Two carbon sp2 orbitals combine with one hydrogen $1 \mathrm{~s}$ orbital each to form C-H bonds: sp2 - 1s $\partial$ bonds (shaded in pink). The remaining carbon sp2 bonding orbital from each carbon is used to form the C-C- bond in ethylene: sp2 - sp2 $\partial$ bond (shaded in blue). One half-occupied, unhybridized $2 \mathrm{Pz}$ orbital left over on each carbon combine to form two new p orbitals (one bonding and one antibonding orbital). Each carbon atom contributes one electron to occupy the bonding orbital to form a: pi bond (shaded in green). This shows the charge is almost neutralizing giving it an insulator characteristic.

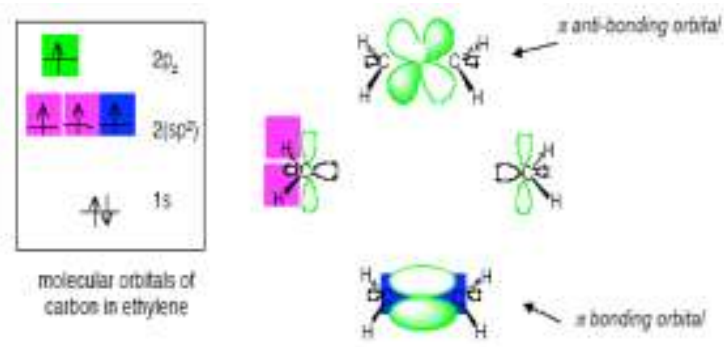

Fig13.Drawn through ACID/ChemSketch Software

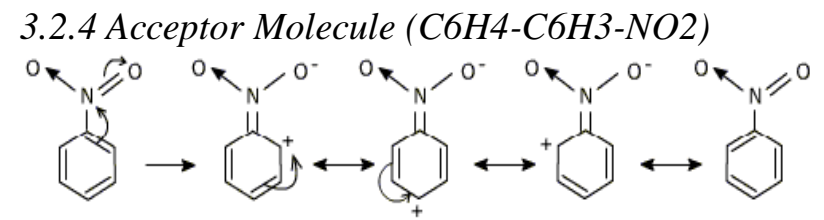

Fig. 14 nitrosobiphenylene (Drawn using ACID/ChemSketch Software)

The nitro group strongly deactivates the benzene ring towards electrophilic substitution.Nitro group is electron withdrawing group and thus causes electron deficiency at ortho and para positrons as is clear from the resonating structures of nitro benzene.

\section{CURRENT VOLATGE CHARACTERSTIC 4.1 DFT and NEGF}

The molecular surface undergoes various voltage range test in the region of insulator, so that multiple donor and acceptor sites can be incorporated. The contacting electrode in both side of donor and acceptor can be gold. Green's function and density functional theories are used to study electron transport characteristics 
through single molecules addressed by two metallic contacts. Each contact is modeled with one nanoscopic end connected to the molecule and one macroscopic end connected to an external potential difference. The method can be applied to any molecular system for which ab initio calculations can be performed. It allows us to determine the molecular orbitals participating in the electron-transfer process, the current-voltage characteristics of the junction, the density of states, and the transmission function, among other properties, providing a fundamental tool for the development of molecular electronics Current-voltage characteristics are in excellent agreement with a break junction experiment and with other ab initio calculations, yielding new insights regarding electron transport through single molecules[5].We use density functional theory (DFT) calculations on molecular junctions consisting of a single molecule between two gold electrodes. The molecules consist of an alkene bridge connecting acceptor-nitro group, donor amine end groups in various combinations. The molecular geometries are optimized and DFT calculates wave functions and eigenstates of the junction .The electron transport properties for the junction are calculated by non-equilibrium Green's function (NEGF) formalism. The current-voltage characteristics for the various molecules in the position of $\mathrm{X}$ and $\mathrm{Y}$ are then compared with rectification is observed for these molecules, particularly for the donor-bridgeacceptor case where the bridge is fixed as alkene. However, at smaller bias rectification is in the opposite direction and is attributed to the lowest unoccupied orbital associated with the acceptor group [6].The nonequilibrium Green's function (NEGF) approach in combination with density functional theory (DFT) was used to investigate the electron transport properties of a single diode molecule that consists of two weakly coupled electron donating $\pi$-system and electron withdrawing $\pi$-system, resembling the conventional $\mathrm{p}-\mathrm{n}$ junctions. The rectification ratio for different sets of X, Y then calculated. The mechanism of the rectification behavior was analyzed in terms of the evolution of molecular energy levels, the change of spatial distribution of molecular orbitals, and the electron transmission spectra. The asymmetric evolutions of the energy levels and the alignment of the states localized on the electro donating and electron withdrawing segments with the applied voltage were found to be essential in generating this rectification behavior for the molecular system [7].Referring to fig.6 ,The nonequilibrium Green's function technique and density functional theory were used to perform quantum-mechanical calculations of electronic transport of single molecular wire .To inspect the substituents effect on the electronic transport properties, both the electron-donating $\left(-\mathrm{NH}_{2}\right)$ and electron-withdrawing $\left(-\mathrm{NO}_{2}\right)$ groups were asymmetrically introduced into the conjugated molecular wire, resembling the semiconductor $p-n$ junction. The results demonstrated the rectification behavior of the molecular wire. The asymmetric evolutions of the energy levels and spatial distributions of the frontier molecular orbitals with the applied voltage are found to be essential in generating this current-voltage asymmetry [8]. Results of this theoretical study are compatible with the assumptions that electron transport occurs through the lowest unoccupied molecular orbital, that the conduction barrier is determined by the molecule chemical potential, and that the molecule becomes charged as the external potential increases. We can explain the nonlinear character of the current-voltage characteristic of the molecule and its temperature dependence [9].

\subsection{Rectifying Diode under Biased Condition and Energy Band}

The band diagram of the mono-molecular diode under zero-bias conditions ahs shown in Fig.15.The three potential barriers exist are one corresponding to the insulating group and two corresponding to the contact between the molecule in donor side and acceptor side attaching to the electrode. These potential barriers provide the required isolation between various parts of the structure. The occupied energy levels in the molecular surface and the Fermi energy level $E_{F}$ are shown. On the left of the central barrier all the pi-type energy levels (HOMO as well as LUMO) are elevated due to the presence of the electron-donating group $\mathrm{X}$ and similarly on the right of the central barrier the energy levels are lowered due to the presence of the electron-withdrawing group Y. This causes a built-in potential to develop across the barrier represented by the energy difference $\Delta \mathrm{E}_{\text {LUMO. For current to flow }}$ electrons must overcome the potential barrier from electron acceptor doped section (S2) to electron donor doped section (S1) and this forms the basis for the formation of the monomolecular rectifying diode. When the molecule forms a dimer or an aggregate, the proximity of the orbitals of the different molecules induces a splitting of the HOMO (Highest Occupied Molecular Orbital) and LUMO (Lowest Unoccupied Molecular Orbital) energy levels. This splitting produces vibrational sublevels which each have their own energy, slightly different from one another. There are as many vibrational sublevels as there are molecules that interact together. When there are enough molecules influencing each other, the number of sublevels is large enough to be perceived as a continuum rather than discrete levels. We no longer consider energy levels, but energy bands. To identify the HOMO and the LUMO of a given molecule, one can find out all the molecular orbitals and fill them with the available electrons, or use a generic ordering of orbitals, and use valence bonding for $\sigma$ type and lone pair orbitals, and molecular orbitals for $\pi$-systems as an approximation.

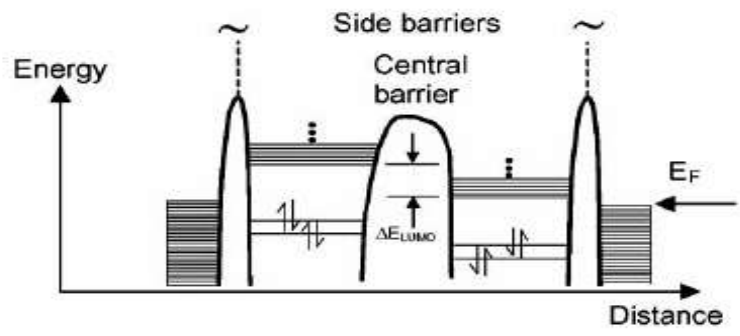

Fig.15.Orbital energy diagram of the polyphenylene monomolecular rectifying diode under zero bias condition.

The energy band diagram under forward bias conditions (left hand contact at higher potential than the right hand contact) is shown in 
Fig.16.Here; electrons are induced to flow by tunneling through the three potential barriers from right to left causing a forward current flow from left to right. The band diagram under reverse bias conditions (left hand contact at lower potential than the right hand contact) is shown in Fig. 17.As a result, electrons from the left contact would try to flow towards the right contact, which is at a higher potential. However, conduction is not possible because the there is still an energy difference between the Fermi energy $E_{F}$ of the left contact and the LUMO energy of the electron donor doped section.

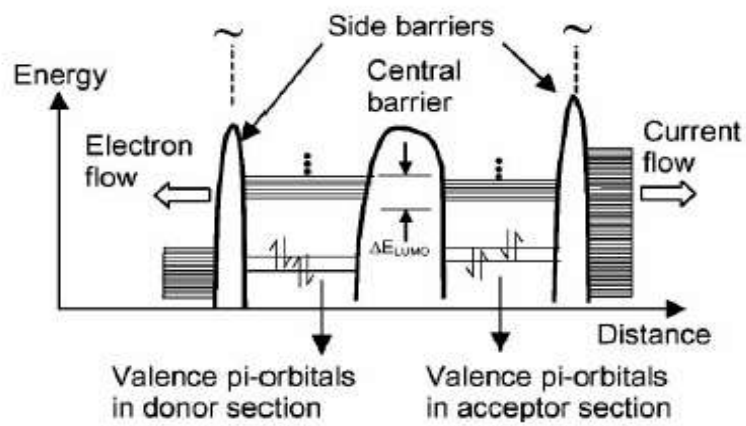

Fig.16-Orbital energy of the polyphenylene monomolecular rectifying diode under forward bias condition

It is assumed that both the applied forward and reverse bias potentials are identical. For a higher reverse bias, however, it is possible for the Fermi energy EF of the left contact to come in resonance with the LUMO energy of the electron donor doped section causing a large current to flow in reverse direction and this is akin to the breakdown condition in a diode. The above monomolecular diode can be used as a building block for realizing molecular digital logic circuits.

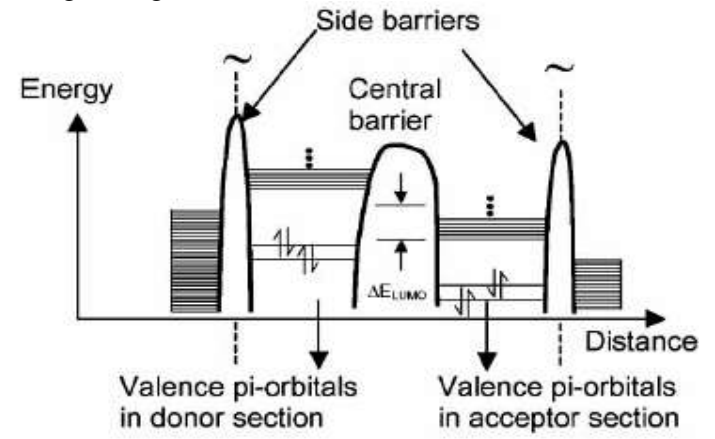

Fig17.Orbital energy of the polyphenylene monomolecular rectifying diode under reverse bias conditions.

The geometric and electronic structures of polyphenylene-based conjugated molecules (donor-spacer-acceptor), potential candidates for molecular rectifying devices, have been investigated theoretically using ab initio quantum mechanical calculations. The individual donor (n-type) and acceptor (p-type) molecular devices have been designed by substituting one or two of the hydrogen atoms of benzene with $-\mathrm{NH} 2$ and $-\mathrm{NO} 2$ functional groups, respectively. The molecular rectifier has been modeled by combining donor and acceptor molecules with various functional groups. The electronic rectification behavior in these molecules has been analyzed from the molecular orbital energy levels and the spatial orientations of the unoccupied molecular orbitals. The results suggest that in such donor-acceptor molecular complexes, while the lowest unoccupied orbital is localized on the acceptor ring, the highest occupied molecular orbital is localized on the donor ring. The approximate potential differences for mono substituted donor-acceptor complexes have to be been estimated for the $-\mathrm{CH} 2-\mathrm{CH} 2-$ spacers. It is found that for the disubstituted rectifier complex, the potential difference increases to $2.76 \mathrm{eV}$ [11]. Similar kind of test has to be performed for the donating group $(\mathrm{X})=\left(-,-\mathrm{OH},-\mathrm{CH}_{3},-\mathrm{CH}_{2} \mathrm{CH}_{3}\right.$, etc. $)$, accepting group $(\mathrm{Y})=(,-\mathrm{CH},-\mathrm{CHO},-\mathrm{NC}$ etc. $)$ and insulating group $\mathrm{R}=\left(-\mathrm{CH}_{2^{-}},-\mathrm{CH}_{2}-\mathrm{CH}_{2^{-}}\right.$, etc. $)$.

\section{CURRENT AND FUTURE DEVELOPMENT}

The current study of DFT and NEGF have to extend more when we will design various gates like approach to AND, OR, XOR, NOT etc. The same principle can apply to for RTD (Resonant Tunneling diode), where the potential barrier rules encounter much. We have still to do more research on computational chemistry aspects to molecular orbitals that affects the electrical properties and we are in way to study more on Schrödinger equation for energy level study in molecular orbitals. The further studies to molecular orbitals for polyatomic molecules, the use of Kopman's theorem and woodwand-hoffman theorem application to energy band of molecular computation need to work out. The Born-Oppenheimer approximation application to molecular computation is to study to find amore robust picture of molecular orbitals that will useful for molecular electronics. We are putting an idea about rectification ratios (RRs), which are crucial for the assessment of the technological usefulness of single molecule junctions as diodes.

\section{CONCLUSION}

The concept of single molecule rectifiers proposed in a theoretical work by Aviram and Ratner was the starting point of the vibrant field of molecular electronics. In the meantime, a built-in asymmetry in the conductance of molecular junctions has been reported at the experimental level. In this scenario, our contribution presents a theoretical representation of molecular orbital concepts and extended to computational chemistry techniques of hybridization that can further explore the orbital features, which will responsible for better flow of electrons in a single molecular wire. This representation carried by classifying the whole single molecule in terms of donor, acceptor and insulator and its behavior in molecular orbital representation. The electronic properties studied from molecular orbital features leads to further research on some more computational chemistry. The same experiment will apply theoretical with many substitute molecules and determines its energy level. We emphasize the importance of the self-consistent charge transfer and voltage drop on the transport characteristics and describe the self-consistent formulation for both device at equilibrium and device out of equilibrium.

\section{REFERENCE}

[1] Kumar M.Jagadesh, February 2007, Molecular Diodes and Applications, Recent Patents on Nanotechnology, Volume1, 
Number1,pp.51-57,

www.bentham.org,http:ingentaconnect.com.

[2] Collier CP, Wong EW, Belohradsky M, Raymo FM, Stoddart JF, Kuekes PJ, Williams RS, Heath JR, 1999, Electronically configurable molecular-based logic gates. Science 1999; 285: 391-394.

[3] Ellenbogen, J.C, 2002, US20026348700B1, Recent Patents on Nanotechnology 2007, 1pp-75-79

[4] Ellenbogen, J.C, 2002, US20026339227B1, Recent Patents on Nanotechnology 2007, 1pp-75-79

[5]. Pedro A. Derosa and Jorge M. Seminario, 2001, Electron Transport through Single Molecules: Scattering Treatment Using Density Functional and Green Function Theories, J. Phys.Chem.B,105(2),pp471-481,DOI:

10.1021/jp003033,American Chemical Society.

[6] M J Ford, R C Hoft, A M McDonagh and M B Cortie, 2008, Rectification in donor-acceptor molecular junctions,JournalofPhysics-Condensed matter, Vol20, Issue37,doi: 10.1088/0953-8984/20/37/374106.

[7] Yanwei Li, Jinhuan Yao, Changjiu Liu and Chuanlu Yang, 2008, Journal of Molecular Structure: THEOCHEM, Volume867, Issues1-3, Pages59-63, doi: 10.1016/j.theochem.2008.07.026

[8] Yanwei Lia, Geping Yina, Jinhuan Yao and Jianwei Zhao, 2008,First-principles study of substituents effect on molecular junctions: Towards molecular rectification, ,Computational Materials Science,Volume 42, Issue 4,Pages 638-642, doi:10.1016/j.commatsci.2007.09.018

[9] Jorge M. Seminario,Angelica G. Zacarias, and James M. Tour, J., 2000, Theoretical Study of a Molecular Resonant Tunneling Diode, Am. Chem. Soc., 122(13),pp30153020,DOI: 10.1021/ja992936h.

[10] Aviram A and Ratner MA, 1974, Molecular Rectfiers.Chem Physics Letter, 29(2):277-283, DOI: 10.1016/00092614(74)85031-1

[11] Chiranjib Majumder, Hiroshi Mizuseki, and Yoshiyuki Kawazoe, 2001, Molecular Scale Rectifier: Theoretical Study. Phys. Chem. A, 2001, 105 (41), pp 9454-9459,DOI: 10.1021/jp011888w, American Chemical Society

[12] C. Joachim, J. K. Gimzewski \& A. Aviram, Electronics using hybrid-molecular and mono-molecular devices, 2000, Nature 408, 541-548, doi:10.1038/35046000

[13] R Stadler, V Geskin and J Cornil , R Stadler ,A theoretical view of unimolecular rectification ,2008 J. Phys.: Condense. Matter 20, doi: 10.1088/0953-8984/20/37/374105.

[14] Yongqiang Xue,Supriyo Datta and Mark A. Ratner, Firstprinciples based matrix Green's function approach to molecular electronic devices: general formalism ,2002,Chemical Physics,Volume281,Issues2-3,Pages151170,Elsevier Science, doi:10.1016/S0301-0104(02)00446-9.

[15] Manas Ranjan Pradhan, E.G.Rajan, 2010, A system engineering approach to molecular electronics, IJCA, Vol-3, Issue8, (14-23), Foundation of Computer Science.
[16] Jorge M.Seminro,Luis E.Cordova, 2003, An ab intito approach to the calculation of current-voltage characteristics of programmable molecular devices, proceedings of the IEEE,Vol.91,11,2003,PP-(1958-1975)

[17] Seminario JM, Zacarias AG, Tour JM, 2000, Theoretical study of a Molecular resonant tunneling diode. J Am Chem Soc, ACS Publication, 122(13): 3015-3020, DOI: 10.1021/ja992936h.

[18] Seika Abe, Masami Hagiya and Takao Nakajima,2004, Code Generation for a DNA Computer by Integer Linear Programming.In:Proceedings of the IEEE Conference on Cybernetics and Intelligent Systems, Singapore, 1-3 December- 2004, 268-273.

[19] Keiichiro Takahashi and Masami Hagiya,2004, On Computation of Minimum Free Energy and Partition Function of Multiple Nucleic Acid Sequences, Forum on Information Science and Technology, 2004, 1:.91-92.

[21] Dr. Richard F.W. Bader, Book-An Introduction to the Electronic Structure of Atoms and Molecules, http://www.chemistry.mcmaster.ca/esam/.

[22] R. S. Mulliken, 1928, "The assignment of quantum numbers for electrons in molecules," Physical Review, 32:186 222.avialablefrom http://prola.aps.org/abstract/PR/v32/i2/p186_1.

[23] J. E. Lennard-Jones, 1929, "The electronic structure of some diatomic molecules," Transactions of the Faraday Society, 25: 668-686.DOI 10.1039/TF9292500668.

[24] Martin Pope and Charles E. Swenberg, 1999, Electronic Processes in Organic Crystals and Polymers, $2^{\text {nd }}$ edition., Oxford Science Publications, Oxford University Press, New York,.

[25] R.S. Mulliken-1966, Spectroscopy, Molecular Orbitals and Chemical Bonding - Nobel Lecture, 131-160.Avaialable at http://130.242.18.21.

[26] Frank Jensen, 1999, Introduction to Computational Chemistry, John Wiley and Sons, 65 - 69, ISBN 0471 98055.

[27] T. V. Rybalova1 and Yu. V. Gatilov1, February, 2010, Molecular pair interaction energy. I. Analysis of supramolecular architecture in the crystals of polyfluorinated enaminoimines and enaminoketones of indan series, Journal of Structural Chemistry, 51(1): 142:148.DOI: 10.1007/s10947-010-0020-4.

[28] N.B.Zhitnev, H.Meng and Z.Bao, 2002,"Conductance of small molecular junctions", Phys.Rev.Lett, vol.88.

[29] J.M.Seminro, C.E.de Las Cruz, and P.A.Deorsa, 2001, A theoretical analysis of metal-molecule contacts, journal America Chemical society, vol123, pp-5616-5617.

[30] J.M.Seminario and P.Politzer, 1995, Modern Density Functional theory: A tool for chemistry, Eds, Amsterdam, The Netherlands: Elsevier.

[31] J.M.Seminario, 1993, Energetics using DFT: comparisions to precise ab initio and experiment, Chem.Phys.Lett, vol.206, pp.547-554. 